\title{
Dietary fibre intake was not associated with a lower risk of colorectal cancer in women
}

\author{
Fuchs CS, Giovannucci EL, Colditz GA, et al. Dietary fiber and the risk of colorectal cancer and adenoma in women. N EnglJ Med \\ 1999 Jan 21;340:169-76.
}

\section{Question}

Is dietary fibre intake associated with a decreased risk of colorectal cancer and adenoma in women?

\section{Design}

Prospective, population based cohort study of women in the Nurses' Health Study with 16 years of follow up.

\section{Setting}

United States.

\section{Participants}

88757 women who were nurses between 30 and 55 years of age in 1976. Exclusion criteria were cancer other than nonmelanoma skin cancer, ulcerative colitis, Crohn's disease, familial polyposis syndrome, implausible high or low scores for total energy intake, or missing food frequency questionnaire data.

\section{Assessment of risk factors}

Nurses completed mailed questionnaires at baseline and every 2 years thereafter. Food frequency questionnaires included information on 61 foods, beverages, and vitamin and mineral supplements. For analysis, nurses were divided into quintiles based on energy adjusted total fibre intake in 1980, 1984, and 1986, with the lowest intake group given the relative risk (RR) of 1.0. Fibre intake was adjusted for total energy intake calculated from dietary intake records of weighed foods. Baseline data also included information on age; weight; height; smoking history; physical activity; aspirin use; colonoscopy or sigmoidoscopy; parental history of colorectal cancer; and red meat, alcohol, fibre, and total energy intake.

\section{Main outcome measure}

Self reported cases of colon or rectal cancer subsequently confirmed by blinded assessment of medical records and pathology reports.

\section{Main results}

787 cases of confirmed invasive colorectal adenocarcinoma were reported. Among the 27530 participants who had an endoscopy, 1012 patients with adenomas of the distal colon and rectum were identified. Median total fibre intake for the lowest and highest quintiles was $9.8 \mathrm{~g} /$ day and $24.9 \mathrm{~g}$ /day, respectively. Based on data collected in 1980, age adjusted and multivariate analyses showed no association between total fibre intake and risk of colorectal cancer (multivariate RR for the highest quintile $0.95,95 \%$ CI 0.73 to 1.25 , p for trend $=0.59$ ). Adjustment of data for combinations of baseline variables, risk factors, or altered or consistent fibre intake during the follow up period did not change this result substantially. The highest quintile of energy adjusted current vegetable fibre intake was associated with an increased risk of colorectal cancer (multivariate RR for the highest quintile 1.35 , CI 1.05 to 1.72 , $\mathrm{p}$ for trend $=0.004$ ).

\section{Conclusion}

Dietary fibre intake was not associated with a decreased risk of colorectal cancer and adenoma in women, but increased vegetable fibre intake was associated with an increased risk of colorectal cancer.

Source of funding: National Institutes of Health

For correspondence: $\operatorname{Dr} C$ S Fuchs, Dana-Farber Cancer Institute, 44 Binney Street, Bostom, MA 02115, USA.Fax +16176325370

\section{Commentary}

This study by Fuchs et al disputes the evidence that a lack of dietary fibre is a contributing factor for colorectal cancer in women. The strength of this study was a large sample of women who did not have other known risk factors for colorectal cancer (eg, family history, previous skin melanomas, or inflammatory bowel diseases). This study also collected and adjusted for extensive self reported demographic baseline data including dietary intake and use of tobacco, medication, and alcohol.

The results showed no decrease in bowel cancer among participants who reportedly included more fibre in their diets. The findings are contrary to common belief and require confirmation in future studies with more precise measures of dietary intake. The study also showed that increased vegetable fibre intake was associated with increased energy adjusted current risk of colorectal cancer; however, vegetable fibre intake was not well defined.

The researchers admit that the use of self reported questionnaires may limit the credibility of their findings. They did, however, attempt to overcome measurement error by using a correction procedure that adjusted RRs and CIs based on a "gold standard" of detailed dietary records of weighed food portions collected for 28 days over a 1 year period by 173 participants.

A more definitive answer to this question can be achieved through systematic review of the literature and statistical combination (meta-analysis) of the find- ings of all relevant prospective studies. In addition, randomised controlled trials in which individuals are randomised to high fibre diets and then followed over the long term for the development of cancer would be the optimal approach to answering this question. Meanwhile, although the study did not provide evidence that dietary fibre reduced the risk of colorectal cancer, nurses should continue to encourage fibre intake for other health reasons such as constipation, and as substitutive foods for high fat and refined sugar foods.

Carol Murphey Garner, RN, EdD Assistant Professor of Nursing

Jacksonville University School of Nursing Jacksonville, Florida, USA 